\title{
Elastic scattering and total reaction cross sections for the ${ }^{8} \mathrm{Li}+{ }^{12} \mathrm{C}$ system
}

\author{
A. Barioni, V. Guimarães, A. Lépine-Szily, R. Lichtenthäler, D. R. Mendes Jr., E. Crema, K. C. C. Pires, M. C. Morais, \\ V. Morcelle, P. N. de Faria, and R. P. Condori \\ Instituto de Física, Universidade de São Paulo, P. O. Box 66318, São Paulo 05389-970, SP, Brazil
}

A. M. Moro

Facultad de Física, Universidad de Sevilla, Apartado 1065, E-41080, Sevilla, Spain

D. S. Monteiro, J. M. B. Shorto, and J. Lubian

Instituto de Fisica, Universidade Federal Fluminense, Niterói, Rio de Janeiro 24210-340, Brazil

M. Assunção

Universidade Federal de São Paulo, Campus Diadema, São Paulo 05389-970, SP, Brazil

(Received 8 July 2009; revised manuscript received 26 August 2009; published 30 September 2009)

\begin{abstract}
The elastic-scattering angular distribution for ${ }^{8} \mathrm{Li}$ on ${ }^{12} \mathrm{C}$ has been measured at $E_{\mathrm{LAB}}=23.9 \mathrm{MeV}$ with ${ }^{8} \mathrm{Li}$ radioactive nuclear beam produced by the Radioactive Ion Beams in Brazil facility. This angular distribution was analyzed in terms of optical-model with Woods-Saxon and double-folding São Paulo potential. The roles of the breakup and inelastic channels were also investigated with cluster folding and deformed potentials, respectively, through coupled-channels calculations. The angular distribution for the proton-transfer ${ }^{12} \mathrm{C}\left({ }^{8} \mathrm{Li},{ }^{9} \mathrm{Be}\right){ }^{11} \mathrm{~B}$ reaction was also measured at the same energy. The spectroscopic factor for the $\left\langle{ }^{9} \mathrm{Be} \mid{ }^{8} \mathrm{Li}+p\right\rangle$ bound system was obtained and compared with shell-model calculations and with other experimental values. Total reaction cross sections for the present system were also extracted from the elastic-scattering analysis. A systematic of the reduced reaction cross sections obtained from the present and published data on ${ }^{6,7,8} \mathrm{Li}$ isotopes on ${ }^{12} \mathrm{C}$ was performed as a function of energy.
\end{abstract}

DOI: 10.1103/PhysRevC.80.034617

PACS number(s): 25.70.-z, 25.60.Bx, 25.60.Je, 21.10.Jx

\section{INTRODUCTION}

Despite the fact that large laboratories are pushing to produce all kinds of exotic and very energetic species of nuclei, some efforts have also been devoted by small laboratories to produce low-energy light radioactive beams. The latter can then be used to investigate low-energy reactions such as elastic scattering, transfer, and breakup, which can provide useful information on the structure of light nuclei near the dripline [1-3]. Moreover, from the theoretical point of view, the investigation of low-energy reactions with light exotic nuclei is of interest, because many of the high-energy approximations that simplify the theoretical treatments, such as classical trajectories and sudden or adiabatic assumptions, are no longer valid. In this way, investigation of low-energy reactions can be considered complementary to the high-energy regime data.

It has been 20 years since the pioneers' experiments with a secondary radioactive ${ }^{8} \mathrm{Li}$ beam have been performed with the radioactive ion beam (RIB) facility installed at the University of Notre Dame [4,5]. At that time a single solenoid was used to separate, collect, and focus the low-energy $\left(E_{\mathrm{LAB}}<20 \mathrm{MeV}\right)$ ${ }^{8} \mathrm{Li}$ secondary beam. Since then, an upgraded system with two solenoids has been installed at University of Notre Dame, USA [6]. More recently a similar system called RIBRAS (Radioactive Ion Beams in Brazil) has been installed at the University of São Paulo, Brazil [7]. With this new facility, we have performed elastic-scattering measurements using a higher-energy ${ }^{8} \mathrm{Li}$ beam $\left(E_{\mathrm{LAB}}>20 \mathrm{MeV}\right)$. Elastic-scattering experiments with ${ }^{8} \mathrm{Li}$ are still of interest due to low binding energy of this lithium isotope. In general, elastic scattering can be an interesting measurement specially when one of the interacting particles is a loosely bound nucleus. It can be considered an effective tool for revealing unusual features in nuclei such as extended halos or neutron skins. Some of these features can be present in the elastic scattering as influence of competing mechanisms and coupled-channels analysis would be required. For instance, due to the lower binding energy of the weakly bound nuclei either direct or sequential breakup can become an important competing mechanism, even at relatively low incident energies. In the analysis of elastic-scattering measurements, breakup effect can be responsible, for instance, for a strong enhancement of the imaginary part of the optical potential, giving rise, sometimes, to what is called "breakup threshold anomaly" [8]. In the case of lithium isotopes such as ${ }^{6} \mathrm{Li}$ and ${ }^{7} \mathrm{Li}$ the influence of breakup effects has been observed in the elastic scattering in several light and medium mass targets, including the ${ }^{12} \mathrm{C}$ target [9-12].

In the analysis of the elastic-scattering angular distribution of ${ }^{6} \mathrm{Li}$ on ${ }^{12} \mathrm{C}$ it has been found necessary to consider a strong reduction of the real part of the folding model potential to describe the data [13], whereas more strongly bound projectiles such as ${ }^{4} \mathrm{He}$ do not require such a renormalization. However, despite the importance of the breakup channel, where an absorptive potential would be required in the analysis, the angular distribution for the ${ }^{6} \mathrm{Li}+{ }^{12} \mathrm{C}$ at several energies has been interpreted by considering a transparent potential that would correspond to an incomplete absorption 
[14]. This contradiction indicates that the interpretation of the elastic-scattering mechanism for a loosely bound nuclei on a light target is not completely understood yet. Moreover, elastic-scattering data of ${ }^{9} \mathrm{Li}$ and ${ }^{11} \mathrm{Li}$ on ${ }^{12} \mathrm{C}$ at intermediate energies show that the nearside/farside interference minimum at forward angles is attenuated in the elastic scattering of ${ }^{11} \mathrm{Li}$ and not for ${ }^{9} \mathrm{Li}$, in contrast to what would be predicted by standard optical-model analysis [15]. As a result, the investigation of elastic scattering of lithium isotopes on ${ }^{12} \mathrm{C}$ still is an interesting subject. ${ }^{8} \mathrm{Li}$, as a neutron-rich nuclei, may also show strong couplings to inelastic or transfer channels in the elastic scattering, because some transfer channels have positive $Q$ value.

In the present work we report on the measurements of an elastic-scattering angular distribution for ${ }^{8} \mathrm{Li}$ on ${ }^{12} \mathrm{C}$ target and an angular distribution for the proton-transfer ${ }^{12} \mathrm{C}\left({ }^{8} \mathrm{Li},{ }^{9} \mathrm{Be}\right){ }^{11} \mathrm{~B}$ reaction at $23.9-\mathrm{MeV}^{8} \mathrm{Li}$ incident energy. The organization of this article is the following: In Sec. II, we describe the experimental procedure used to measure the new data reported here. The optical-model and double-folding model used to describe the elastic-scattering data are discussed in Sec. III, where a CDCC (continuum discretized coupledchannels) and an usual coupled-channels (including only the inelastic channels) calculation are presented. The FR-DWBA analysis of the proton transfer reaction is reported in Sec. IV. Comparison of spectroscopic factor obtained from the present experiment and shell-model calculations is presented in Sec. V. The energy dependence of the reaction cross section for the lithium isotopes on carbon, including the present reported data and the previously published data, are investigated and the results are discussed in Sec. VI. The final discussions and conclusions are left for Sec. VII.

\section{DESCRIPTION OF THE EXPERIMENT}

We have performed elastic scattering and proton-transfer angular distribution measurements using radioactive secondary ${ }^{8} \mathrm{Li}$ beam impinged on a ${ }^{12} \mathrm{C}$ at São Paulo University, São Paulo, Brazil. In this experiment the ${ }^{8} \mathrm{Li}$ secondary beam was produced with the RIBRAS radioactive nuclear beam system [7]. In this system, the ${ }^{8} \mathrm{Li}$ radioactive beam was produced in a primary ${ }^{9} \mathrm{Be}$ target by the neutron transfer reaction ${ }^{9} \mathrm{Be}\left({ }^{7} \mathrm{Li},{ }^{8} \mathrm{Li}\right)$. The ${ }^{9} \mathrm{Be}$ production target had a thickness of $16 \mu \mathrm{m}$ and was mounted in an ISO chamber just before the first solenoid. For more details on the description of this RIB system and on the recent scientific program with RIBRAS, see Ref. [16]. The primary ${ }^{7} \mathrm{Li}$ beam was obtained from a 8-MV Pelletron accelerator with intensity between 200 and $500 \mathrm{nAe}$ and energy of $27 \mathrm{MeV}$. After crossing the production target, the ${ }^{7} \mathrm{Li}$ primary beam is suppressed in a tungsten Faraday cup that measures its intensity. A current integrator measures the total charge incident on the primary target during a run. The secondary ${ }^{8} \mathrm{Li}$ beam produced in the forward direction is collected and focused within an angular range of $2^{\circ}<\theta<6^{\circ}$ by the first solenoid of the double-superconducting solenoids of the RIBRAS facility. The magnetic field of the first solenoid is adjusted to focus the secondary beam of interest in the ISO250 scattering chamber. A system of blocks and collimators, located just after the solenoid, prevent particles with different magnetic rigidity from reaching the scattering target. Although the RIBRAS system has two solenoids, we used only one in this experiment. The energy of the secondary ${ }^{8} \mathrm{Li}$ beam was $23.9 \mathrm{MeV}$ at the center of the carbon target with an average intensity of about $5 \times 10^{4} \mathrm{pps}$. Some beam contaminants $\left({ }^{4} \mathrm{He}\right.$, ${ }^{6} \mathrm{He}$, and ${ }^{7} \mathrm{Li}$ ) with the same magnetic rigidity as ${ }^{8} \mathrm{Li}$ were also present but did not produce reaction products with mass $A=8$ in the same range of energy of the scattered ${ }^{8} \mathrm{Li}$ particles of interest.

The scattered ${ }^{8} \mathrm{Li}$ particles and reaction products were detected by an array of $\Delta E-E$ Si telescopes. The measurements were performed with three telescopes covering the range of angles from $20^{\circ}$ to $60^{\circ}$ in the laboratory system. The telescopes consisted of $20-\mu \mathrm{m} \mathrm{Si} \Delta E$ detectors with an area of $300 \mathrm{~mm}^{2}$ backed by $1000-\mu \mathrm{m}$-thick Si $E$ detectors. The telescopes had rectangular apertures that subtended a solid angle of about $12 \mathrm{msr}$.

The target used in the measurements was composed of a polyethylene plastic $\left(\mathrm{CH}_{2}\right), 1.6 \mathrm{mg} / \mathrm{cm}^{2}$ thick, mounted together with a thin gold target $350 \mu \mathrm{g} / \mathrm{cm}^{2}$ thick. The idea was to perform simultaneously the elastic-scattering measurement of ${ }^{8} \mathrm{Li}$ on carbon and gold targets, where the elastic scattering on gold target is to be used to obtain the overall normalization, because at these energies it is expected to be essentially Rutherford. The elastic-scattering angular distribution was measured from $20^{\circ}$ to $60^{\circ}$ in the laboratory system in $5^{\circ}$ steps. The $5^{\circ}$ step has been chosen because the angular aperture of the collimators in front of the detectors was also about $\pm 3^{\circ}$. Because the cross sections in the angular interval covered by these detectors could vary up to one order of magnitude, the average detection angle was determined by a Monte Carlo simulation, which took into account the collimator size in front of the detectors, the secondary beam spot size on the secondary target $(4 \mathrm{~mm})$, the secondary beam divergence, and the angular distribution in the range of the detector aperture (Rutherford on gold and calculated in an iterative way for the ${ }^{12} \mathrm{C}$ target). This correction is particularly important for the most forward angles.

Reaction products were identified using a two-dimensional [ $\left.\Delta E \times E_{\text {total }}\right]$ plot. A selected particle identification spectrum for the experiment is shown in Fig. 1. In this plot, the scattered ${ }^{8} \mathrm{Li}$ beam particles, the ${ }^{4} \mathrm{He}$ and ${ }^{7} \mathrm{Li}^{2+}$ beam contaminants, and the reaction products are shown and could be identified. The ${ }^{8} \mathrm{Li}$ energy spectra were obtained by selecting and projecting the ${ }^{8} \mathrm{Li}$ region in the $\left[\Delta E \times E_{\text {total }}\right]$ plot. The experimental resolution of $500 \mathrm{KeV}$ obtained from the full width at half maximum of the peak corresponding to the ${ }^{8} \mathrm{Li}$ scattering only in gold target was enough to separate the ${ }^{8} \mathrm{Li}$ scattered from the carbon and gold targets. To obtain the elastic yields, a line-shape analysis using Gaussian forms for the peaks was considered for the spectra obtained at forward angles. In the energy spectra obtained at $20^{\circ}$, where the separation of the ${ }^{8} \mathrm{Li}$ peak corresponding to elastic scattering on carbon and gold could be more critical, a measurement with separate targets was also performed and it was consistent with the measurement with both targets together. For the most backward angles measurements, the ${ }^{8} \mathrm{Li}$ yields were determined based on the expected energy of the group. The inelastic scattering to 


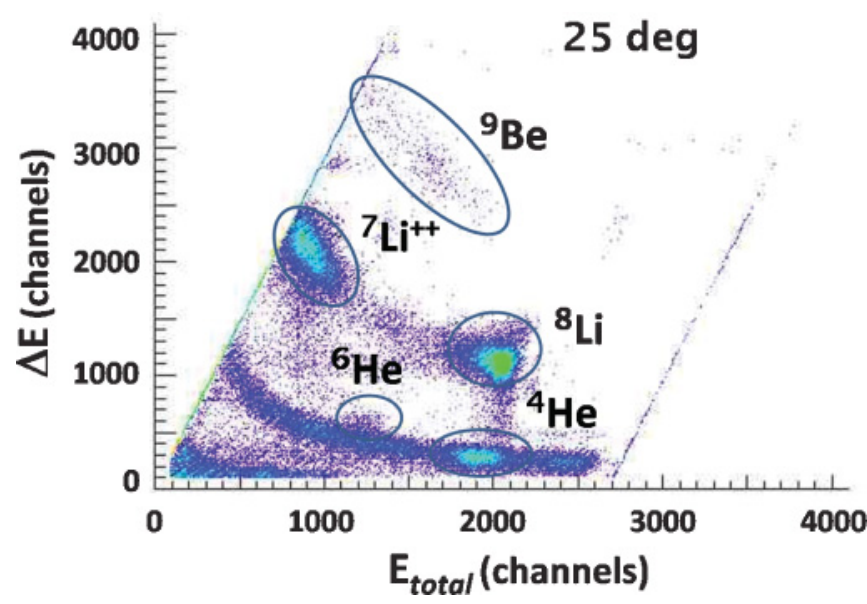

FIG. 1. (Color online) Selected particle identification spectrum $\left[\Delta E \times E_{\text {total }}\right]$ for the interaction of ${ }^{8} \mathrm{Li}+\left(\mathrm{CH}_{2}+\mathrm{Au}\right)$ at $E_{\mathrm{LAB}}=$ $23.9 \mathrm{MeV}$ measured at $25^{\circ}$. The elastic scattering $\left({ }^{8} \mathrm{Li}\right)$ and reaction product ${ }^{9} \mathrm{Be}$ are indicated as are the ${ }^{4} \mathrm{He},{ }^{7} \mathrm{Li}^{++}$, and ${ }^{6} \mathrm{He}$ contamination in the secondary beam.

the first excited state of ${ }^{8} \mathrm{Li}\left(E_{x}=0.980 \mathrm{MeV}\right)$ would be expected for the gold target but it would be very small for the carbon target and the experimental resolution would be enough to separate the inelastic from the elastic-scattered ${ }^{8} \mathrm{Li}$ particles. However, there is no strong indication of inelastic scattering in the energy spectra.

The experimental elastic-scattering angular distribution obtained is shown in Fig. 2. The uncertainties in the differential cross sections were estimated considering the statistical uncertainty in the yields and the systematic uncertainties in the target thickness (10\%) and the secondary beam intensity $(10 \%)$.

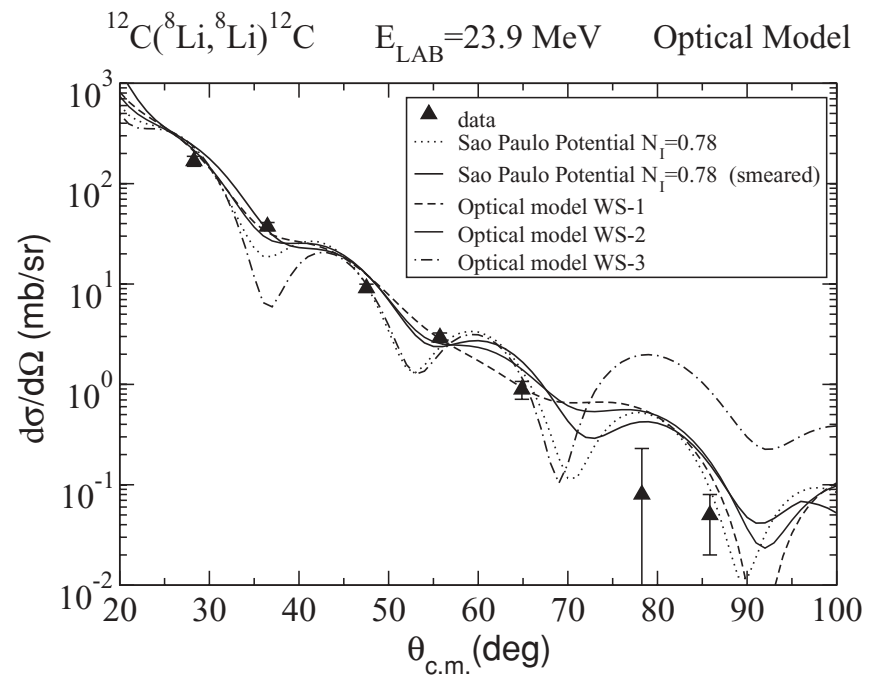

FIG. 2. The differential cross sections for elastic scattering $\left({ }^{12} \mathrm{C}\left({ }^{8} \mathrm{Li},{ }^{8} \mathrm{Li}\right){ }^{12} \mathrm{C}\right)$ at $23.9-\mathrm{MeV}$ incident laboratory energy. The curves are optical-model calculations with the sets of parameters listed in Table I. The curve for the São Paulo potential is smeared out by the angular aperture of the detectors.

\section{A. Optical-model calculations}

Considering the standard procedure, we first analyzed the elastic-scattering angular distribution with the optical-model (OM) calculations using volume-type Woods-Saxon (WS) and double-folding nuclear potentials (plus Coulomb potentials due to uniform charged spheres). The WS optical potentials parameters used are listed in Table I. All calculations were performed with the code FRESCO [17]. The results of the OM calculations can be seen in Fig. 2. The curve indicated as the São Paulo (SP) potential corresponds to OM calculations using the São Paulo potential [18], which is a double-folding potential with energy dependence and nonlocality correction. In this potential, the used average diffuseness for the charge and matter distributions are $a=0.53 \mathrm{fm}$ and $a=0.56$ $\mathrm{fm}$, respectively. Within the context of the systematics for the densities, the SP potential has no adjustable parameters. The imaginary part of the potential has the same form factor as the real part and a normalization of $N_{I}=0.78$.

The OM calculations with the SP potential show more oscillations than the elastic-scattering data and we smeared out the angular distributions by the range of the angular aperture of the detectors $\left(\Delta \Theta=10^{\circ}\right.$ in the center of mass). As one can see, the comparison with the data is improved by smearing the calculated angular distributions. Also, including a spinorbit term in the optical-model $\left(V_{\mathrm{SO}}=7.0 \mathrm{MeV}\right)$ calculations did not change the results. In conclusion, the SP potential reproduces quite well the absolute normalization, which is of some interest, considering that this folding-model potential has no free parameters. The potentials parameters WS- 2 and WS-3 in Table I were obtained from an average of energy-dependent potentials used to describe ${ }^{7} \mathrm{Li}+{ }^{12} \mathrm{C}$ and ${ }^{6} \mathrm{Li}+{ }^{12} \mathrm{C}$ elastic scattering from $E_{\mathrm{LAB}}=4.5$ to about $100 \mathrm{MeV}$ [19]. We can see in Fig. 2 that the potential WS-2, which is derived from the elastic scattering of ${ }^{7} \mathrm{Li}+{ }^{12} \mathrm{C}$ at $24 \mathrm{MeV}$, is more suitable to describe the ${ }^{8} \mathrm{Li}+{ }^{12} \mathrm{C}$ system than the potential WS-3 derived from the ${ }^{6} \mathrm{Li}+{ }^{12} \mathrm{C}$ system. It is important to emphasize that there was no attempt to adjust the parameters to fit the data. The potentials WS- 1 and WS- 2 give a good description of the elastic-scattering data at forward angles and they were used later for the entrance channel in the proton transfer reaction analysis.

\section{B. CDCC calculations}

In this section we analyze the same elastic-scattering angular distribution as in the previous sections but now in terms of the CDCC formalism. The motivation to perform such a calculation is to investigate more explicitly the role of the continuum in the scattering.

In the CDCC calculation the interaction between the projectile ${ }^{8} \mathrm{Li}$ and the target ${ }^{12} \mathrm{C}$ is written as the sum of the interactions $\mathrm{U}\left({ }^{7} \mathrm{Li}+{ }^{12} \mathrm{C}\right)$ and $\mathrm{U}\left(n+{ }^{12} \mathrm{C}\right)$. For the $\mathrm{U}\left({ }^{7} \mathrm{Li}+{ }^{12} \mathrm{C}\right)$ potential we considered the parameters listed in Table I taken from Ref. [20] and for the $\mathrm{U}\left(n+{ }^{12} \mathrm{C}\right)$ we have adopted the parameters given by a global nucleon-nucleus parametrization from Ref. [21], see Table I. Another important ingredient in the CDCC calculation is the potential used to obtain the bound-state wave function of the ${ }^{8} \mathrm{Li}$ nucleus, 
TABLE I. Optical-model potential parameters. Radii are given by $R_{x}=r_{x} \times\left(A_{P}^{1 / 3}+A_{T}^{1 / 3}\right)$. The depths are in MeV and the radius and diffuseness are in $\mathrm{fm}$.

\begin{tabular}{|c|c|c|c|c|c|c|c|c|c|}
\hline System & $V$ & $r_{V}$ & $a_{V}$ & $W_{V}$ & $r_{W}$ & $a_{W}$ & $r_{C}$ & & Reference \\
\hline${ }^{8} \mathrm{Li}+{ }^{12} \mathrm{C}$ (WS-1) & 159.0 & 0.63 & 0.73 & 7.20 & 1.38 & 0.850 & 1.25 & & ${ }^{7} \mathrm{Li}+{ }^{12} \mathrm{C} 34 \mathrm{MeV}[20]$ \\
\hline${ }^{8} \mathrm{Li}+{ }^{12} \mathrm{C}(\mathrm{WS}-2)$ & 167.0 & 0.60 & 0.80 & 9.57 & 1.31 & 0.720 & 1.25 & & ${ }^{7} \mathrm{Li}+{ }^{12} \mathrm{C} 24 \mathrm{MeV}$ [19] \\
\hline${ }^{8} \mathrm{Li}+{ }^{12} \mathrm{C}(\mathrm{WS}-3)$ & 158.4 & 0.752 & 0.79 & 7.27 & 1.31 & 0.62 & 1.25 & & ${ }^{6} \mathrm{Li}+{ }^{12} \mathrm{C} 24 \mathrm{MeV}$ [19] \\
\hline${ }^{9} \mathrm{Be}+{ }^{11} \mathrm{~B}(\mathrm{WS}-4)$ & 60.0 & 0.96 & 0.60 & 32.6 & 1.18 & 0.60 & 1.1 & & ${ }^{9} \mathrm{Be}+{ }^{12} \mathrm{C} 26 \mathrm{MeV}[25]$ \\
\hline${ }^{8} \mathrm{Li}+{ }^{11} \mathrm{~B}$ (core-core) & 159.0 & 0.63 & 0.73 & 7.20 & 1.38 & 0.850 & 1.25 & & ${ }^{7} \mathrm{Li}+{ }^{12} \mathrm{C} 34 \mathrm{MeV}[20]$ \\
\hline${ }^{7} \mathrm{Li}+{ }^{12} \mathrm{C}(\mathrm{CDCC})$ & 159.0 & 0.63 & 0.73 & 7.20 & 1.38 & 0.850 & 1.25 & & ${ }^{7} \mathrm{Li}+{ }^{12} \mathrm{C} 34 \mathrm{MeV}[20]$ \\
\hline $\begin{array}{l}n+{ }^{12} \mathrm{C}(\mathrm{CDCC}) \\
n+{ }^{7} \mathrm{Li}(\mathrm{CDCC})\end{array}$ & $\begin{array}{l}54.45 \\
45.30\end{array}$ & $\begin{array}{l}1.128^{\mathrm{a}} \\
1.25^{\mathrm{a}}\end{array}$ & $\begin{array}{l}0.57 \\
0.52\end{array}$ & $\begin{array}{c}W_{S} \\
8.28\end{array}$ & $\begin{array}{c}a_{w} \\
0.50\end{array}$ & $\begin{array}{l}V_{\text {SO }} \\
5.5 \\
6.0\end{array}$ & $\begin{array}{l}a_{\text {SO }} \\
0.57 \\
0.52\end{array}$ & $\begin{array}{c}r_{W}=r_{\mathrm{SO}} \\
1.128^{\mathrm{a}} \\
1.25^{\mathrm{a}}\end{array}$ & {$[21]$} \\
\hline
\end{tabular}

${ }^{\mathrm{a}} R_{x}=r_{x} \times\left(A_{T}^{1 / 3}\right)$.

$\mathrm{U}\left(n+{ }^{7} \mathrm{Li}\right)$. For this binding potential we assumed the parameters listed in Table I with a spin-orbit term. The depth of this potential was determined to reproduce the binding energy of the ${ }^{7} \mathrm{Li}+n={ }^{8} \mathrm{Li}$ system $(\mathrm{BE}=2.033 \mathrm{MeV})$. The continuum spectrum was divided into eight bins with equal energy width in the range of 0 to $8 \mathrm{MeV}$ in excitation energy of ${ }^{8} \mathrm{Li}$. All the possible couplings between the ${ }^{7} \mathrm{Li}$ core, $J^{\pi}=3 / 2^{-}$, and the valence neutron with $s, p$, and $d$ waves for the relative motion of $n+{ }^{7} \mathrm{Li}$ were considered. This CDCC calculation were also performed with the computer code FRESCO, where the convergence was achieved with a matching radius of $50 \mathrm{fm}$ and a maximum total angular momentum of $L_{\mathrm{MAX}}=50$. This procedure has been successfully used to study the elastic and breakup channels of many reactions involving weakly bound nuclei. Here we want to verify the importance of the breakup channel of ${ }^{8} \mathrm{Li}$ into ${ }^{7} \mathrm{Li}+n$ in the description of the ${ }^{8} \mathrm{Li}+{ }^{12} \mathrm{C}$ elastic scattering. The results of such calculations can be seen in Fig. 3. For comparison we considered the elastic-scattering

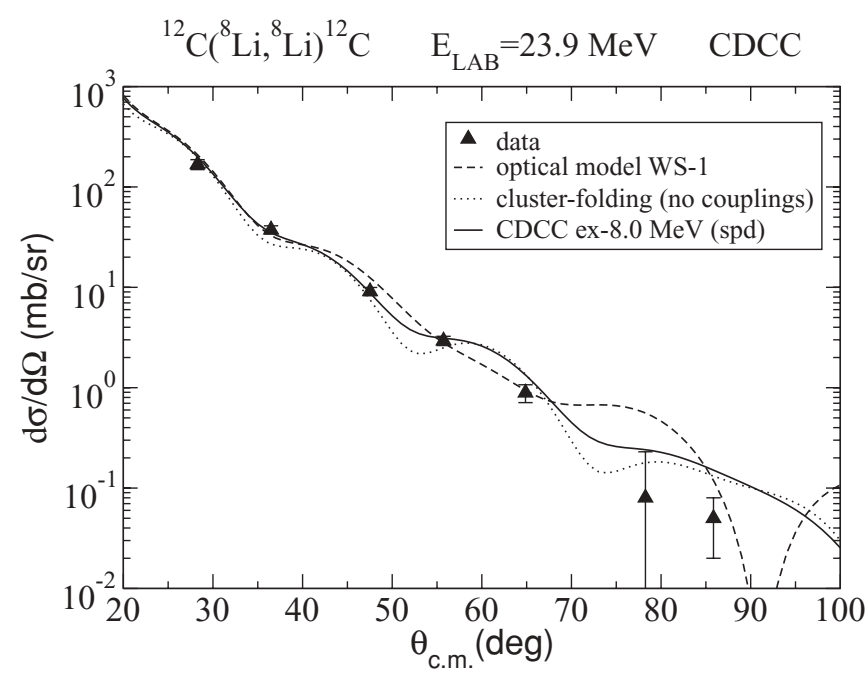

FIG. 3. The differential cross sections for elastic scattering ${ }^{12} \mathrm{C}\left({ }^{8} \mathrm{Li},{ }^{8} \mathrm{Li}\right){ }^{12} \mathrm{C}$ at $23.9-\mathrm{MeV}$ incident laboratory energy. The dashed curve is an optical-model potential with parameters given by WS in Table I. The dotted curve is the cluster-folding parametrization of the interaction without the couplings to the continuum while the solid line is the result of the coupling. calculation with the pure optical model (dashed lines), with the cluster-folding parametrization, as described above, for the ${ }^{8} \mathrm{Li}+{ }^{12} \mathrm{C}$ interaction, and without and with the couplings to the continuum (dotted and solid lines, respectively). Because these calculated curves did not show strong oscillations they were not smeared out by the angular range of the detectors. As one can see in Fig. 3, the cluster folding parametrization and the pure optical potential model give an equivalent description of the data. Also a similar description of the data is achieved if couplings to the continuum is taken into account. Based on these calculations we can then conclude that the effect of the breakup channel in this elastic-scattering angular distribution is small, as it would be expected for a light system with low $\mathrm{Z}$ target.

\section{Coupled-channels calculations}

Another approach to analyze the elastic-scattering angular distribution data is to consider the collective model for ${ }^{8} \mathrm{Li}$ and ${ }^{12} \mathrm{C}$. With such deformed potential we can generate the excited state of ${ }^{8} \mathrm{Li}$ and ${ }^{12} \mathrm{C}$ and perform coupled-channels calculations to verify the effect of the collective excitations in the elastic scattering. The Coulomb and nuclear matrix elements were obtained from the experimental value of the reduced transition probability $B(E 2)$ and the deformation length $\delta$, respectively.

The energy resolution of the present experiment was barely sufficient to separate elastic events from inelastic scattering to the $0.98-\mathrm{MeV}$ first excited state of ${ }^{8} \mathrm{Li}$. However, due to the poor statistics, the elastic events at most backward angles could be contaminated with inelastic events. We estimated the inelastic cross section and also the effect of this channel to the elastic with coupled-channels calculation using the code FRESCO. The calculations were performed for a $2^{+} \rightarrow 1^{+}$transition of ${ }^{8} \mathrm{Li}$ projectile assumed to be members of the $k=1$ rotational band with parameters taken from Ref. [22] corresponding to $B(E 2)=30 e^{2} \mathrm{fm}^{4}$ and a projectile deformation length of $1.75 \mathrm{fm}$. Also we considered the $0^{+} \rightarrow 2^{+}$transition of ${ }^{12} \mathrm{C}$ with quadrupole deformation $\beta=0.582$ from Ref. [23]. Both projectile and target deformations were applied to the real part of the nuclear potential, given by the São Paulo potential. Despite the large values, the predicted cross sections for the 


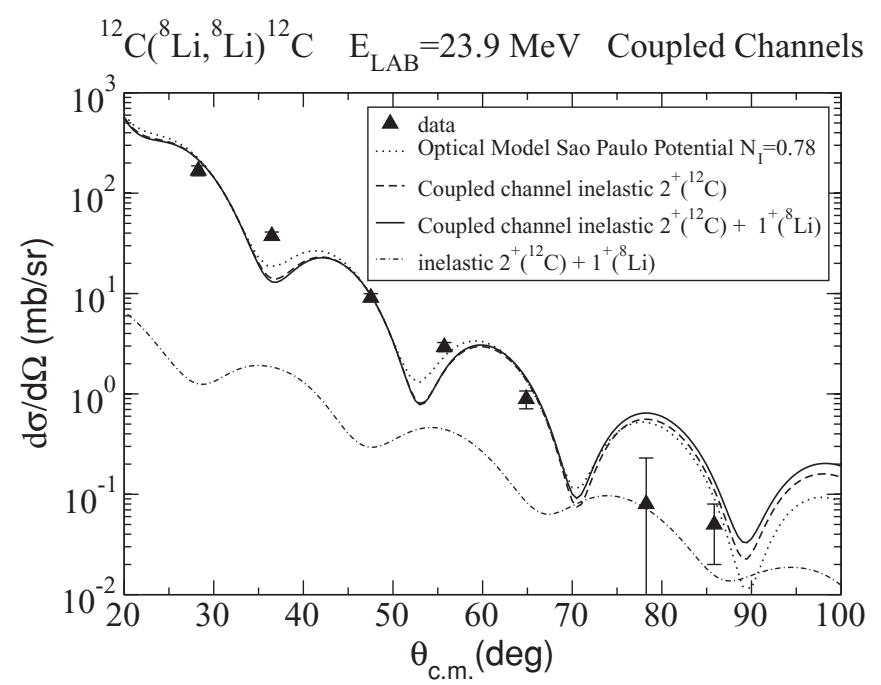

FIG. 4. The differential cross sections for elastic scattering ${ }^{12} \mathrm{C}\left({ }^{8} \mathrm{Li},{ }^{8} \mathrm{Li}\right){ }^{12} \mathrm{C}$ at $23.9-\mathrm{MeV}$ incident laboratory energy. The dotted curve is an optical-model calculation with SP potential. The dashed curve is the coupled-channels calculation with only the $2^{+}$first excited state in ${ }^{12} \mathrm{C}$ and the solid line is the coupled-channels calculation including also the $1^{+}$first excited state in ${ }^{8} \mathrm{Li}$. The dotted-dashed curve is the prediction of the inelastic scattering including both first excited states in ${ }^{8} \mathrm{Li}$ and ${ }^{12} \mathrm{C}$.

inelastic cross sections are relevant only for the most backward angles. By considering the couplings to the inelastic channel, the calculated elastic-scattering distributions are changed only a small amount by an optical-model calculation that ignores inelastic excitation. The results of these calculations, without smearing them out by the angular aperture of the detectors, can be seen in Fig. 4.

\section{PROTON-TRANSFER REACTION, ${ }^{12} \mathrm{C}\left({ }^{8} \mathrm{Li},{ }^{9} \mathrm{Be}\right){ }^{11} \mathrm{~B}$}

In addition to the elastic scattering, some other nuclear reactions were also identified for the ${ }^{8} \mathrm{Li}+{ }^{12} \mathrm{C}$ system measured at $23.9 \mathrm{MeV}$. Among them, the positive $Q_{g g}$ value $=0.931 \mathrm{MeV}$ proton-transfer ${ }^{12} \mathrm{C}\left({ }^{8} \mathrm{Li},{ }^{9} \mathrm{Be}\right){ }^{11} \mathrm{~B}$ reaction. The experimental angular distribution obtained for this reaction is shown in Fig. 5. The differential cross sections for this transfer process are very small (in the range of 0.1 to $1.0 \mathrm{mb} / \mathrm{sr}$ ), which made the measurements and analysis difficult due to the limited secondary beam intensity. Also, exactly due to this limited secondary beam intensity we have considered the cross sections only for the first four angles, namely $20^{\circ}, 25^{\circ}, 30^{\circ}$, and $35^{\circ}$ in the laboratory system. The uncertainties in the differential cross sections were estimated considering the statistical uncertainty in the yields and the systematic uncertainties in the target thickness $(10 \%)$, the secondary beam intensity $(10 \%)$, and solid angles ( $10 \%$ to $15 \%)$.

This proton transfer angular distribution has been analyzed in terms of a FR-DWBA (finite-range distorted-wave Born approximation) calculation also using the code FRESCO. For the entrance $\left({ }^{8} \mathrm{Li}+{ }^{12} \mathrm{C}\right)$ and exit $\left({ }^{9} \mathrm{Be}+{ }^{11} \mathrm{~B}\right)$ channels we used the double-folding São Paulo potential calculated for

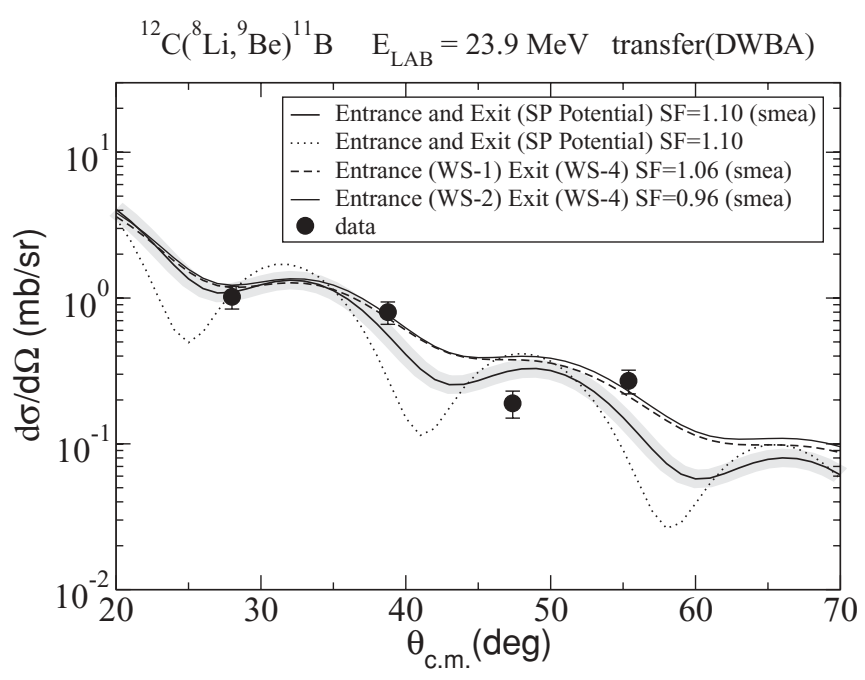

FIG. 5. Angular distribution for the ${ }^{12} \mathrm{C}\left({ }^{8} \mathrm{Li},{ }^{9} \mathrm{Be} g s\right){ }^{11} \mathrm{~B}_{\mathrm{gs}}$ protontransfer reaction at $23.9 \mathrm{MeV}$ incident energy. The curves are indicated in the legend inset. The gray thick line corresponds to experimental error of the spectroscopic factor in the FR-DWBA calculation with SP potential. The curves are smeared out by the angular aperture of the detectors.

each system with the imaginary part normalized as $N_{I}=0.78$. For the core-core potential, $\mathrm{U}\left({ }^{8} \mathrm{Li}+{ }^{11} \mathrm{~B}\right)$, we used the one listed in Table I from Ref. [20]. A different choice for this potential such as WS-3 in the Table I produced no change in the final result. The bound-state wave functions were generated with Woods-Saxon potentials and geometric parameters $r=1.25 \mathrm{fm}$ and $a=0.65 \mathrm{fm}$, with the depths of the potentials adjusted to give the correct separation energies. The proton-transfer ${ }^{12} \mathrm{C}\left({ }^{8} \mathrm{Li},{ }^{9} \mathrm{Be}\right){ }^{11} \mathrm{~B}$ reaction can proceed by transferring the proton from ${ }^{12} \mathrm{C}_{\mathrm{gs}}\left(J^{\pi}=0^{+}\right)$to either $p_{3 / 2}$ or $p_{1 / 2}$ orbital in ${ }^{8} \mathrm{Li}_{\mathrm{gs}}\left(J^{\pi}=2^{+}\right)$, leaving both ${ }^{9} \mathrm{Be}$ and ${ }^{11} \mathrm{~B}$ in its ground state $J^{\pi}=\frac{3}{2}^{-}$. However, the contribution of the $p_{1 / 2}$ orbital admixture in the ${ }^{9} \mathrm{Be}$ ground state is found to be $11 \%$ of that $p_{3 / 2}$ based in the shell-model calculation by Cohen and Kurath [24]. FR-DWBA calculations gave angular distributions for these two contributions very similar in shape, differing only in absolute value. Thus, because we could not experimentally distinguish them we instead constrained the spectroscopic factor of the $p_{1 / 2}$ orbital to be $11 \%$ of that for the $p_{3 / 2}$ state and considered a coherent sum of these two contributions. The results for such a FR-DWBA calculation with an SP potential for the entrance and exit channel is also shown in Fig. 5. As one can see, the calculation agrees reasonably with the data within experimental uncertainties. We also considered Woods-Saxon potentials for the entrance and exit channels. Two combinations of potentials were considered for the entrance(exit) channel, WS-1(WS-4) and WS-2(WS-4). The potential WS-4 used as the potential for the exit channel was derived from the ${ }^{9} \mathrm{Be}+{ }^{12} \mathrm{C}$ elastic-scattering data [25]. For the core-core potential $\mathrm{U}\left({ }^{8} \mathrm{Li}+{ }^{11} \mathrm{~B}\right)$, we used a potential derived from the analysis of ${ }^{7} \mathrm{Li}+{ }^{12} \mathrm{C}$ [20]. The results for the FR-DWBA calculation with these Woods-Saxon potentials describe relatively well the experimental angular distribution, as can be seen in Fig. 5 . 


\section{SPECTROSCOPIC FACTOR AND SHELL-MODEL CALCULATIONS}

To extract the spectroscopic factor from the data we compared the experimental angular distribution for the proton transfer reaction ${ }^{12} \mathrm{C}\left({ }^{8} \mathrm{Li},{ }^{9} \mathrm{Be}\right){ }^{11} \mathrm{~B}$ with a FR-DWBA calculation. In this procedure we actually obtain the product of the two spectroscopic factors for the two vertices, $\left({ }^{8} \mathrm{Li},{ }^{9} \mathrm{Be}\right)$ and $\left({ }^{12} \mathrm{C},{ }^{11} \mathrm{~B}\right)$. The values obtained were $C^{2} S\left({ }^{9} \mathrm{Be}_{\mathrm{gs}}\right) \times$ $C^{2} S\left({ }^{12} \mathrm{C}_{\mathrm{gs}}\right)=2.56$ for the analysis with Sao Paulo potential and Woods-Saxon potentials. Thus, to obtain the spectroscopic factor for the $\left\langle\left.{ }^{9} \mathrm{Be}_{\mathrm{gs}}\right|^{8} \mathrm{Li}_{\mathrm{gs}}+p\right\rangle$ bound system, the spectroscopic factor for $\left\langle\left.{ }^{12} \mathrm{C}_{\mathrm{gs}}\right|^{11} \mathrm{~B}_{\mathrm{gs}}+p\right\rangle$ must be known. For the $\left({ }^{12} \mathrm{C},{ }^{11} \mathrm{~B}\right)$ vertex, the spectroscopic factor was taken to be $\mathrm{C}^{2} \mathrm{~S}\left({ }^{12} \mathrm{C}_{\mathrm{gs}}\right)=2.33 \pm 0.35$, which is the average of values from $\left({ }^{3} \mathrm{He}, d\right)$ and $\left(d,{ }^{3} \mathrm{He}\right)$ studies [26-30]. By normalizing the FR-DWBA calculation to the experimental data, a spectroscopic factor of $\mathrm{C}^{2} \mathrm{~S}\left({ }^{9} \mathrm{Be}_{\mathrm{gs}}\right)=1.10 \pm 0.25$ was obtained for the $\left\langle{ }^{9} \mathrm{Be}_{\mathrm{gs}} \mid{ }^{8} \mathrm{Li}_{\mathrm{gs}}+p\right\rangle$ bound system. The uncertainty in the spectroscopic factor is estimated to be about $18 \%$ due to the uncertainties in the experimental data at forward angles and $15 \%$ due to the spectroscopic factor of the other vertex, $\left\langle{ }^{12} \mathrm{C}_{\mathrm{gs}} \mid{ }^{11} \mathrm{~B}_{\mathrm{gs}}+p\right\rangle$. By considering Woods-Saxon potentials to describe the entrance and exit channel, a smaller spectroscopic factor is obtained, $\mathrm{C}^{2} \mathrm{~S}\left({ }^{9} \mathrm{Be}_{\mathrm{gs}}\right)=1.06$ and $\mathrm{C}^{2} \mathrm{~S}\left({ }^{9} \mathrm{Be}_{\mathrm{gs}}\right)=0.96$, for the two choices of potentials considered. The spectroscopic factor obtained for the $\left\langle{ }^{9} \mathrm{Be}_{\mathrm{gs}} \mid{ }^{8} \mathrm{Li}_{\mathrm{gs}}+p\right\rangle$ bound system in the present analysis is compared with other experimental values in Table II. Our result agrees within the experimental error with the values obtained from $\left(d,{ }^{3} \mathrm{He}\right)[31],(t, \alpha)[32]$ but it is almost twice higher than the one obtained from the $(d, p)$ reaction [33] and lower than the value obtained with the ${ }^{9} \mathrm{Be}\left({ }^{8} \mathrm{Li},{ }^{9} \mathrm{Be}\right){ }^{8} \mathrm{Li}$ reaction [34]. In principle, as the spectroscopic factor reflects the nuclear structure, it should be independent of reactions and incident energies. However, instead of a constant value, different experimental spectroscopic factors are obtained from different reactions. These fluctuations could be in part due to the use of different potential models. Thus, a consistent choice of potential parameters could be a useful way to extract structure information. To avoid such inconsistencies many authors are using standard geometric parameters $\left(r_{0}=1.25 \mathrm{fm}\right.$ and $a=0.65 \mathrm{fm}$ ) for the bound-state potentials. A consistent systematic investigation on neutron spectroscopic factor has been performed by M. B. Tsang et al. [35] and a similar systematic for proton spectroscopic factor would be welcome. Here, although similar potential parameters for the bound state have been used for the proton transfer reactions, the spectroscopic factors vary by almost a factor of 2.5.

The value for $\mathrm{C}^{2} \mathrm{~S}\left({ }^{9} \mathrm{Be}_{\mathrm{gs}}\right)$ obtained with the present proton transfer reaction is also compared in Table II with the predictions of the old shell-model calculation by Cohen and Kurath [24] and with a new calculation using the WBT interaction of Warbuton and Brown [36] with the code OXBASH [37]. In this new calculation, $0 \mathrm{p} 0 \mathrm{~h}$ and $2 \mathrm{p} 2 \mathrm{~h}$ excitations $(0 \hbar \omega+2 \hbar \omega)$ and spsdf model space have been taken into account for ${ }^{8} \mathrm{Li}$ and ${ }^{9} \mathrm{Be}$. The results for both $\left(p_{1 / 2}\right.$ and $\left.p_{3 / 2}\right)$ components of the overlap $\left\langle{ }^{9} \mathrm{Be} \mid{ }^{8} \mathrm{Li}+p\right\rangle$ are displayed in Table II. The experimental value of $\mathrm{C}^{2} \mathrm{~S}_{\exp }\left({ }^{9} \mathrm{Be}_{\mathrm{gs}}\right)=1.10(25)$ agrees better with the old shell-model calculation by Cohen and Kurath, $\mathrm{C}^{2} \mathrm{~S}_{\text {calc }}\left({ }^{9} \mathrm{Be}_{\mathrm{gs}}\right)=1.00$ than with the new one, $\mathrm{C}^{2} \mathrm{~S}_{\mathrm{calc}}\left({ }^{9} \mathrm{Be}_{\mathrm{gs}}\right)=$ 0.87 . However, if we take into account that what we obtain from the experimental angular distribution is the product of the two spectroscopic factors, $\mathrm{C}^{2} \mathrm{~S}\left({ }^{9} \mathrm{Be}_{\mathrm{gs}}\right) \times \mathrm{C}^{2} \mathrm{~S}\left({ }^{12} \mathrm{C}_{\mathrm{gs}}\right)$, then the agreement between the experimental value, 2.56 , and the theoretical prediction with the new shell-model calculation, 2.57 , is excellent.

\section{TOTAL REACTION CROSS SECTIONS}

Another way to investigate the role of breakup of weakly bound or exotic nuclei on a specific reaction mechanism is to plot the cross section for weakly and tightly bound nuclei on the same target nucleus as a function of energy. In this way different breakup threshold energies are involved and the role of breakup can be investigated. Here we performed such analysis by considering the total reaction cross section.

The total reaction cross sections for the ${ }^{8} \mathrm{Li}+{ }^{12} \mathrm{C}$ system were obtained from the elastic-scattering angular distribution analysis using the double-folding SPP (São Paulo potential). In this analysis the normalization parameter for the real part of the potential $N_{R}=1.0$ were kept fixed. We, however, allowed a variation of the imaginary strength parameter, $N_{I}$, where the value for each energy has been adjusted to fit the elasticscattering angular distributions. For the ${ }^{8} \mathrm{Li}+{ }^{12} \mathrm{C}$ system we also considered the data taken at $14 \mathrm{MeV}$ by the Notre DameMichigan group [22].

TABLE II. Spectroscopic factors $C^{2} S$.

\begin{tabular}{|c|c|c|c|}
\hline & $\begin{array}{l}\text { Shell-model calculation } \\
\qquad\left(p_{3 / 2}+p_{1 / 2}\right)\end{array}$ & $\begin{array}{c}\text { Other } \\
\text { experimental values }\end{array}$ & $\begin{array}{c}\text { This work } \\
\left(p_{3 / 2}+p_{1 / 2}\right)\end{array}$ \\
\hline$\left\langle\left.{ }^{9} \mathrm{Be}_{\mathrm{gs}}\right|^{8} \mathrm{Li}_{\mathrm{gs}}+p\right\rangle \quad J^{\pi}=3 / 2^{-}$ & $1.00^{\mathrm{a}}, 0.87^{\mathrm{b}}$ & $\begin{array}{c}0.64^{\mathrm{c}}, 1.00^{\mathrm{d}}, \\
1.059^{\mathrm{e}}, 1.67(31)^{\mathrm{f}}\end{array}$ & $1.10(25)$ \\
\hline$\left\langle{ }^{12} \mathrm{C}_{\mathrm{gs}} \mid{ }^{11} \mathrm{~B}_{\mathrm{gs}}+p\right\rangle$ & $2.843^{\mathrm{a}}, 2.95^{\mathrm{b}}$ & $2.33(35)^{\mathrm{g}}$ & \\
\hline $\begin{array}{l}\text { a Shell-model calculation from } \\
\text { b Shell-model calculation with } \\
{ }^{\mathrm{c}} \text { From the } \mathrm{d}\left({ }^{8} \mathrm{Li}, n\right){ }^{9} \mathrm{Be} \text { reaction } \\
{ }^{\mathrm{d}} \text { From the }{ }^{9} \mathrm{Be}\left(d,{ }^{3} \mathrm{He}\right){ }^{8} \mathrm{Li} \text { react } \\
{ }^{\mathrm{e}} \text { From the }{ }^{9} \mathrm{Be}(t, \alpha){ }^{8} \mathrm{Li} \text { reaction } \\
{ }^{\mathrm{f}} \text { From the }{ }^{9} \mathrm{Be}\left({ }^{8} \mathrm{Li},{ }^{9} \mathrm{Be}\right){ }^{8} \mathrm{Li} \text { reac } \\
{ }^{\mathrm{g}} \text { Average of } 2.00[26], 2.44 \text { [27 }\end{array}$ & $\begin{array}{l}\text { Cohen and Kurath [24]. } \\
\text { WBT interaction (OXBASH } \\
\text { at } 40 \mathrm{MeV} \text { [33]. } \\
\text { tion at } 52 \mathrm{MeV} \text { [31]. } \\
\text { at } 15 \mathrm{MeV}[32] . \\
\text { tion at } 27 \mathrm{MeV}[34] . \\
\text { ], } 2.98[28] 2.125[29], 2 .\end{array}$ & {$[30]$} & \\
\hline
\end{tabular}


TABLE III. Reaction cross section from the analysis of the elastic-scattering angular distributions of all systems indicated.

\begin{tabular}{|c|c|c|c|c|c|c|c|}
\hline System & $E_{\text {c.m. }}$ & $N_{I}$ & $\sigma(\mathrm{mb})$ & $\chi^{2}$ & $E_{\mathrm{red}}$ & $\sigma_{\text {red }}$ & Ref. \\
\hline \multirow[t]{2}{*}{${ }^{8} \mathrm{Li}+{ }^{12} \mathrm{C}$} & 8.4 & 0.56 & 1197 & 3.9 & 2.00 & 65.0 & {$[22]$} \\
\hline & 14.3 & 1.35 & 1523 & 28.1 & 3.41 & 82.8 & Present \\
\hline \multirow[t]{4}{*}{${ }^{7} \mathrm{Li}+{ }^{12} \mathrm{C}$} & 4.7 & 1.12 & 731 & 0.3 & 1.11 & 41.4 & [42] \\
\hline & 5.7 & 1.18 & 917 & 1.1 & 1.33 & 51.9 & $"$ \\
\hline & 7.6 & 0.91 & 1105 & 8.0 & 1.77 & 62.6 & $"$ \\
\hline & 9.5 & 0.90 & 1211 & 5.2 & 2.21 & 68.6 & $"$ \\
\hline \multirow[t]{5}{*}{${ }^{6} \mathrm{Li}+{ }^{12} \mathrm{C}$} & 6.0 & 0.89 & 901 & 4.7 & 1.37 & 53.4 & [40] \\
\hline & 7.3 & 0.36 & 976 & 10.4 & 1.67 & 57.8 & \\
\hline & 8.7 & 0.39 & 1059 & 21.0 & 1.98 & 62.8 & [39] \\
\hline & 16.0 & 0.99 & 1323 & 26.6 & 3.65 & 78.4 & $"$ \\
\hline & 20.0 & 0.80 & 1305 & 54.4 & 4.56 & 77.4 & $"$ \\
\hline${ }^{6} \mathrm{He}+{ }^{12} \mathrm{C}$ & 12.0 & 0.78 & 1384 & 16.1 & 4.11 & 82.1 & [41] \\
\hline \multirow[t]{2}{*}{${ }^{4} \mathrm{He}+{ }^{12} \mathrm{C}$} & 9.8 & 3.00 & 1106 & 41.8 & 3.15 & 73.6 & [38] \\
\hline & 13.5 & 3.00 & 1168 & 322.3 & 4.36 & 77.7 & \\
\hline \multirow[t]{10}{*}{${ }^{11} \mathrm{~B}+{ }^{12} \mathrm{C}$} & 5.4 & 3.00 & 352 & 8.3 & 0.81 & 17.3 & [43] \\
\hline & 6.5 & 1.13 & 585 & 1.1 & 0.98 & 28.7 & $"$ \\
\hline & 7.6 & 1.36 & 822 & 0.8 & 1.14 & 40.4 & $”$ \\
\hline & 8.1 & 2.27 & 977 & 1.2 & 1.22 & 47.9 & $"$ \\
\hline & 8.6 & 2.26 & 1049 & 0.9 & 1.29 & 51.5 & $"$ \\
\hline & 10.5 & 2.35 & 1265 & 1.6 & 1.58 & 62.1 & $"$ \\
\hline & 11.5 & 1.48 & 1256 & 2.2 & 1.73 & 61.7 & $"$ \\
\hline & 14.6 & 1.09 & 1354 & 23.8 & 2.20 & 66.5 & $"$ \\
\hline & 20.9 & 1.14 & 1505 & 24.1 & 3.14 & 73.9 & $"$ \\
\hline & 26.1 & 1.32 & 1526 & 1.3 & 3.93 & 74.9 & $"$ \\
\hline \multirow[t]{2}{*}{${ }^{9} \mathrm{Be}+{ }^{12} \mathrm{C}$} & 11.4 & 0.87 & 1250 & 3.5 & 2.08 & 65.5 & [25] \\
\hline & 14.9 & 1.07 & 1395 & 12.7 & 2.71 & 73.1 & \\
\hline
\end{tabular}

The obtained values for the total reaction cross sections for the ${ }^{8} \mathrm{Li}+{ }^{12} \mathrm{C}$ system are listed in Table III together with the total reaction cross sections obtained for some other systems involving light nuclei projectile on carbon target: ${ }^{4} \mathrm{He}+{ }^{12} \mathrm{C}$ [38], ${ }^{6} \mathrm{Li}+{ }^{12} \mathrm{C}[39,40],{ }^{6} \mathrm{He}+{ }^{12} \mathrm{C}[41],{ }^{7} \mathrm{Li}+{ }^{12} \mathrm{C}$ [42], ${ }^{9} \mathrm{Be}+{ }^{12} \mathrm{C}$ [25], and ${ }^{11} \mathrm{~B}+{ }^{12} \mathrm{C}$ [43]. Among these systems we have combinations with weakly bound nuclei projectiles, such as the lithium isotopes ${ }^{6,7,8} \mathrm{Li},{ }^{9} \mathrm{Be}$, and ${ }^{6} \mathrm{He}$, and more tightly bound nuclei projectiles, such as ${ }^{4} \mathrm{He}$ and ${ }^{11} \mathrm{~B}$. For consistency, we have reanalyzed the elastic-scattering angular distribution for all these systems. All the elastic-scattering angular distributions were fitted using the SPP, allowing for the variation of $N_{I}$, considering only the forward angles (up to $60^{\circ}-70^{\circ}$ at center of mass) where the elastic-scattering mechanism is predominant. Different values of $N_{I}$ were obtained for different systems at different energies. A good description of the angular distributions for ${ }^{11} \mathrm{~B}+{ }^{12} \mathrm{C}$ elastic scattering has been achieved with the double-folding analysis but a better description than that shown in the original articles was not obtained for the other systems.

To compare the total reaction cross sections for the different systems we used the procedure suggested in Ref. [44], where the cross sections are divided by $\left(A_{P}^{1 / 3}+A_{T}^{1 / 3}\right)^{2}$ and the centerof-mass energy by $Z_{P} Z_{T} /\left(A_{P}^{1 / 3}+A_{T}^{1 / 3}\right)$, with $Z_{P}\left(Z_{T}\right)$ and $A_{P}\left(A_{T}\right)$ standing for the charge and mass of the projectile

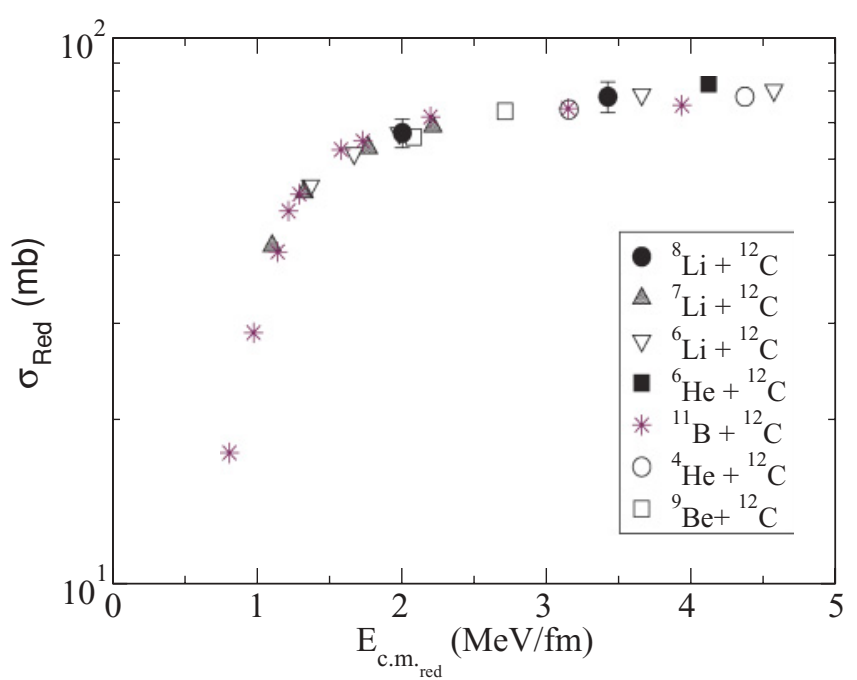

FIG. 6. The reduced reaction cross sections for the ${ }^{8} \mathrm{Li}+{ }^{12} \mathrm{C}$ system obtained in this work together with reduced reaction cross sections of the other lithium isotopes and some weakly bound and tightly bound projectiles on ${ }^{12} \mathrm{C}$.

(target), respectively. In this way, the geometrical effects are removed and the eventual anomalous values of the reduced radii $r_{0}$, which should be related to the physical processes to be investigated, are not washed out. These reduced parameters are also presented in Table III. Figure 6 shows the results of the reduced total reaction cross sections, $\sigma_{\text {red }}$, for all the systems listed in Table III plotted as a function of the reduced energy. Similar comparison have been performed for systems with heavier targets. It has been observed that for heavier targets such as ${ }^{58} \mathrm{Ni}$, ${ }^{64} \mathrm{Zn}$, or even ${ }^{209} \mathrm{Bi}$, where the Coulomb breakup predominates over the nuclear breakup, larger reduced total reaction cross sections have been obtained at energies around the Coulomb barrier for exotic nuclei $\left({ }^{6} \mathrm{He}\right.$ and $\left.{ }^{8} \mathrm{~B}\right)$ followed by the weakly bound nuclei $\left({ }^{6} \mathrm{Li},{ }^{7} \mathrm{Li},{ }^{8} \mathrm{Li}\right.$, and $\left.{ }^{9} \mathrm{Be}\right)$, and then the tightly bound nuclei $\left({ }^{16} \mathrm{O}\right.$ and $\left.{ }^{4} \mathrm{He}\right)$ that produces the smallest total reaction cross section $[1,45]$. This has been explained as due to the effect of breakup channel. In particular, for total reaction cross section for ${ }^{8} \mathrm{Li}$ on a heavy target, due to the intense long-range Coulomb field, the transfer and breakup cross sections were found to account for most of the total reaction cross section [46]. Even for the combination of light nuclei on medium mass targets, where the Coulomb field is not so intense, transfer and breakup cross sections were found to be much more important than the fusion cross section at energies above the Coulomb barrier [44,47]. For a target as light as ${ }^{12} \mathrm{C}$, the breakup channel seems to be not as important as for heavier targets and the reduced reaction cross sections for weakly bound lithium isotopes as well as for helium isotopes $\left({ }^{4} \mathrm{He}\right.$ and $\left.{ }^{6} \mathrm{He}\right)$ and for a tightly bound nuclei $\left({ }^{11} \mathrm{~B}\right)$ follow the same trend. These results are in agreement with the CDCC calculations performed in Sec. II B, where it was shown that coupling to the continuum states was of minor relevance.

\section{SUMMARY}

In this work, we reported the measurements and analysis of the elastic-scattering angular distribution of ${ }^{8} \mathrm{Li}$ on ${ }^{12} \mathrm{C}$ and the 
proton transfer reaction ${ }^{12} \mathrm{C}\left({ }^{8} \mathrm{Li},{ }^{9} \mathrm{Be}\right){ }^{11} \mathrm{~B}$ at $E_{\mathrm{LAB}}=23.9 \mathrm{MeV}$. The measured elastic-scattering angular distribution could be reproduced quite well by using a conventional Woods-Saxon and double-folding optical potentials. To verify the effects of the breakup (continuum) and inelastic contributions to the elastic data, we have performed coupled-channels calculations with and without the coupling to the continuum and with the cluster folding and collective model to describe the ${ }^{8} \mathrm{Li}$ nucleus. These calculations indicated that neither breakup nor inelastic channels are relevant in the description of the elastic-scattering data.

Also, a FR-DWBA analysis has been performed for the proton transfer reaction ${ }^{12} \mathrm{C}\left({ }^{8} \mathrm{Li},{ }^{9} \mathrm{Be}\right){ }^{11} \mathrm{~B}$ at $E_{\mathrm{LAB}}=23.9 \mathrm{MeV}$, where the spectroscopic factor for the $\left\langle{ }^{9} \mathrm{Be}_{\mathrm{gs}} \mid{ }^{8} \mathrm{Li}_{\mathrm{gs}}+p\right\rangle$ bound system was obtained. The spectroscopic factor obtained was compared with shell-model calculations and also with experimental values from different reactions.
The reduced reaction cross sections as a function of reduced energy for the lithium isotope as well as for the halo Borromean ${ }^{6} \mathrm{He}$ nucleus and tightly bound ${ }^{4} \mathrm{He}$ and ${ }^{11} \mathrm{~B}$ on the light target ${ }^{12} \mathrm{C}$ have the same behavior, indicating that for light systems the effects of the binding energy through the breakup on the reaction cross sections is very small.

\section{ACKNOWLEDGMENTS}

The authors thank the Fundação de Amparo a Pesquisa do Estado de São Paulo (FAPESP 2003/10099-2), Fundação de Amparo a Pesquisa do Estado do Rio de Janeiro (FAPERJ), the Conselho Nacional de Pesquisa e Desenvolvimento (CNPq), and Projeto de ação complementaria internacional Sevilla-São Paulo (PCI2006-A7-0654) for financial support. The author A. Barioni thanks the Coordenação de Aperfeiçoamento de Pessoal de Nivel Superior (CAPES) for the financial support.
[1] E. Benjamim, A. Lepine-Szily, D. R. Mendes Jr., R. Lichtenthaler, V. Guimarães, P. R. S. Gomes, L. C. Chamon, M. Hussein, A. M. Moro, and A. Arazi, Phys. Lett. B647, 30 (2007).

[2] V. Guimarães, J. J. Kolata, D. Peterson, P. Santi, R. H. WhiteStevens, S. M. Vincent, F. D. Becchetti, M. Y. Lee, T. W. ODonnell, D. A. Roberts, and J. A. Zimmerman, Phys. Rev. Lett. 84, 1862 (2000).

[3] V. Guimarães, R. Lichtenthaler, O. Camargo, A. Barioni, M. Assunção, J. J. Kolata, H. Amro, F. D. Becchetti, Hao Jiang, E. F. Aguilera, D. Lizcano, E. Martines-Quiroz, and H. Garcia, Phys. Rev. C 75, 054602 (2007).

[4] J. J. Kolata, A. Morsad, X. J. Kong, R. E. Warner, F. D. Becchetti, W. Z. Liu, D. A. Roberts, and J. W. Janecke, Nucl. Instrum. Methods B 40-41, 503 (1989).

[5] F. D. Becchetti, W. Z. Liu, D. A. Roberts, J. W. Janecke, J. J. Kolata, A. Morsad, X. J. Kong, and R. E. Warner, Phys. Rev. C 40, R1104 (1989).

[6] F. D. Becchetti et al., Nucl. Instrum. Methods A 505, 377 (2003).

[7] R. Lichtenthaler et al., Eur. Phys. J. A 25, S-01, 733 (2005).

[8] M. S. Hussein, P. R. S. Gomes, J. Lubian, and L. C. Chamon, Phys. Rev. C 73, 044610 (2006).

[9] Y. Sakuragi, Phys. Rev. C 35, 2161 (1987).

[10] Y. Sakuragi, M. Kamimura, and K. Katori, Phys. Lett. B205, 204 (1988)

[11] J. M. Figueira et al., Phys. Rev. C 73, 054603 (2006).

[12] J. M. Figueira et al., Phys. Rev. C 75, 017602 (2007).

[13] G. R. Satchler and W. G. Love, Phys. Rep. 55, 183 (1979).

[14] F. Michel and S. Ohkubo, Phys. Rev. C 72, 054601 (2005).

[15] D. Peterson, J. J. Kolata, P. Santi, J. von Schwarzenberg, D. Bazin, and B. M. Sherrill, Phys. Rev. C 67, 014601 (2003).

[16] R. Lichtenthaler et al., AIP Conf. Proc. 1139, 76 (2009).

[17] I. J. Thompson, Comput. Phys. Rep. 7, 167 (1988); www.fresco.org.uk.

[18] L. C. Chamon, B. V. Carlson, L. R. Gasques, D. Pereira, C. De Conti, M. A. G. Alvarez, M. S. Hussein, M. A. Candido Ribeiro, E. S. Rossi Jr., and C. P. Silva, Phys. Rev. C 66, 014610 (2002).

[19] M. F. Vineyard, J. Cook, K. W. Kemper, and M. N. Stephens, Phys. Rev. C 30, 916 (1984).
[20] J. Cook, M. N. Stephens, and K. W. Kemper, A. K. Abdallah, Phys. Rev. C 33, 915 (1986).

[21] B. A. Watson, P. P. Sinch, and R. E. Seguel, Phys. Rev. 182, 977 (1969).

[22] R. J. Smith, J. J. Kolata, K. Lamkin, A. Morsad, F. D. Becchetti, J. A. Brown, W. Z. Liu, J. W. Janecke, D. A. Roberts, and R. E. Warner, Phys. Rev. C 43, 2346 (1991).

[23] S. Raman, C. W. Nestor Jr., and P. Tikkanen, At. Data Nucl. Data Tables 78, 1 (2001).

[24] S. Cohen and D. Kurath, Nucl. Phys. A101, 1 (1967).

[25] L. Jarczyk, J. Okolowicz, and A. Strzalkowski, Nucl. Phys. A316, 139 (1979).

[26] D. W. Devins, D. L. Friesel, W. P. Jones, A. C. Attard, I. D. Svalbe, V. C. Officer, R. S. Henderson, B. M. Spicer, and G. G. Shute, Aust. J. Phys. 32, 323 (1979).

[27] M. Gaillard, R. Bouche, L. Feuvrais, P. Gaillard, A. Guichard, M. Gusakow, J. L. Leonhardt, and J. R. Pizzi, Nucl. Phys. A119, 161 (1968).

[28] F. Hinterberger, G. Mairle, U. Schmidt-Rohr, P. Turek, and G. J. Wagner, Nucl. Phys. A106, 161 (1968).

[29] G. M. Reynolds, D. E. Rundquist, and R. M. Poichar, Phys. Rev. C 3, 442 (1971).

[30] G. S. Mutchler, D. Rendic, D. E. Velkley, W. E. Sweeney Jr., and G. C. Philips, Nucl. Phys. A172, 469 (1971).

[31] U. Schwinn, G. Mairle, G. J. Wagner, and Ch. Ramer, Z. Phys. A 275, 241 (1975).

[32] G. B. Liu and H. T. Fortune, Phys. Rev. C 38, 1985 (1988).

[33] J. Su, Z. H. Li, B. Guo, W. P. Liu, X. X. Bai, S. Zeng, G. Lian, S. Q. Yan, B. X. Wang, and Y. B. Wang, Chin. Phys. Lett. 23, 55 (2006).

[34] O. Camargo et al., Phys. Rev. C 78, 034605 (2008).

[35] M. B. Tsang, Jenny Lee, and W. G. Lynch, Phys. Rev. Lett. 95, 222501 (2005).

[36] E. K. Warburton and B. A. Brown, Phys. Rev. C 46, 923 (1992).

[37] B. A. Brown, A. Etchegoyen, and W. D. Rae, the Oxford Buenos Aires MSU shell model code. MSUCL Report No. 524 (1988).

[38] Y.-X. Yang and Q.-R. Li, Nucl. Phys. A732, 3 (2004).

[39] D. E. Trcka, A. D. Frawley, K. W. Kemper, D. Robson, J. D. Fox, and E. G. Myers, Phys. Rev. C 41, 2134 (1990). 
[40] J. E. Poling, E. Norbeck, and R. R. Carlson, Phys. Rev. C 13, 648 (1976).

[41] M. Milin, S. Cherubini, T. Davinson, A. Di Pietro, P. Figuera, D. Miljanic, A. Musumarra, A. Ninane, A. N. Ostrowski, M. G. Pellegriti, A. C. Shotter, N. Soic, C. Spitaleri, and M. Zadro, Nucl. Phys. A730, 285 (2004).

[42] V. V. Parkar, K. Mahata, S. Santra, S. Kailas, A. Shrivastava, K. Ramachandran, A. Chatterjee, V. Jha, and P. Singh, Nucl. Phys. A792, 187 (2007).
[43] L. Jarczyk, B. Kamys, A. Strzalkowski, A. Szczurek, M. Godlewski, J. Lang, R. Müller, and J. Sromicki, Phys. Rev. C 31, 12 (1985).

[44] P. R. S. Gomes, J. Lubian, I. Padron, and R. M. Anjos, Phys. Rev. C 71, 017601 (2005).

[45] J. J. Kolata and E. F. Aguilera, Phys. Rev. C 79, 027603 (2009).

[46] E. F. Aguilera et al., Phys. Rev. C 63, 061603(R) (2001).

[47] P. R. S. Gomes et al., Phys. Lett. B601, 20 (2004). 